\title{
Mechanisms of selective feature adaptation
}

\author{
W. A. AINSWORTH \\ Department of Communication, University of Keele, Keele, Staffordshire, ST5 5BG, England
}

\begin{abstract}
There are two mechanisms which might explain the shift in perceptual boundaries between phonemes which is brought about by repeated stimulation. A feature detector might become "fatigued" so that it responded less vigorously or less reliably, or a feature detector might become "retuned" so that it responded optimally to a different value of some parameter. A simple theoretical description of each mechanism is given, and the predictions are compared with the result of an experiment. The best agreement between theory and experiment was obtained by assuming the fatigue hypothesis to be correct.
\end{abstract}

Eimas and Corbit (1973) have postulated the existence of feature detectors as part of the human speech recognition mechanism. They have suggested that the existence of such detectors can be demonstrated by repeated stimulation so that they become fatigued and less sensitive to further stimulation.

In particular, Eimas and Corbit have postulated that there are two feature detectors tuned to different values of voice onset time (VOT), the time between the release burst in a stop consonant and the start of laryngeal pulsing. Lisker and Abramson (1967) have shown that this dimension is appropriate for classifying stop consonants as voiced (negative or short values of VOT) or voiceless (longer values of VOT). In the normal situation, it is supposed that the feature detector that is tuned to the value of VOT closest to that of the incoming stimulus responds more vigorously than the other, signaling to the higher region of the analyzing system the presence of the feature that this detector represents.

If stimuli having VOT values intermediate between those appropriate for voiced and those-appropriate for voiceless stop consonants are presented for identification, the position of the perceptual boundary along the VOT dimension can be determined. Eimas and Corbit (1973) were able to demonstrate that if the "voiced" detector was fatigued by many repetitions of a voiced stop consonant, the position of the boundary shifted away from the "voiceless" end of the VOT dimension towards the "voiced" end. It was found that if the adapting stimulus was $/ \mathrm{b} /$ the $/ \mathrm{b} /-/ \mathrm{p} /$ boundary shifted towards $/ \mathrm{b} /$, whereas if it was $/ p /$ the boundary shifted in the opposite direction. Similarly, the $/ d /-/ t /$ boundary could be shifted with $/ d /$ or $/ t /$. Furthermore, it was found that $/ d /$ shifted the $/ b /-/ p /$ boundary towards $/ b /$, and $/ b /$ shifted the $/ d /-/ t /$ boundary towards $/ \mathrm{d} /$. These cross-series experiments showed that the detectors must respond to features of the sounds rather than to complete syllables.

Eimas, Cooper, and Corbit (1973) have taken the view that the detectors respond to linguistic features of the stimuli. These linguistic features correspond approximately to the "distinctive features" of Jacobson, Fant, and Halle (1963). Further evidence for the existence of linguistic feature detectors has been provided by Cooper (1974), who demonstrated perceptual boundary shifts along a dimension related to place of articulation.

Bailey (1974) has suggested that the adaptation takes place at the level of acoustic feature analysis, rather than at the higher linguistic levels. Acoustic features are features of the sound itself, such as formant frequencies or the time intervals between events, whereas linguistic features are more abstract and refer to the language to which the syllable belongs rather than to physical attributes of the sound. Bailey has shown that it is only when the relevant features of the sounds occupy the same region of the spectrum that adaptation takes place.

Ainsworth (1975), Diehl (1975), and Ganong (1975) have attempted to distinguish between acoustic feature adaptation and linguistic feature adaptation. In each of these experiments, an attempt was made to devise adaptor syllables which had linguistic features in common with the test syllables but did not share acoustic features with them. Significant shifts were found when the adaptor and test syllables shared acoustic features, but smaller shifts occurred when the adaptor and test syllables shared linguistic, but not acoustic, features, suggesting that adaptation might take place at both levels. Pisoni (1975), however, has demonstrated that the linguistic feature analyzers are not as general as required by distinctive feature theory. Consonant-vowel syllables do not adapt vowel-consonant syllables, and vice versa.

Ades (1974) has attempted to determine the site of adaptation in the brain. By stimulating one ear with the adapter syllable and the other with the test syllable, he was able to show that adaptation occurred centrally. On the other hand, he also showed, by simultaneous adaptation of different ears, that each hemisphere of the brain could be adapted in a different direction. This suggests that the specialized 
speech analyzers, which are thought to reside in the left hemisphere of the brain (Studdert-Kennedy \& Shankweiler, 1970), are not necessary in order to demonstrate selective adaptation effects.

All of the foregoing experiments have been performed with synthesized syllables as stimuli. Although these syllables may be quite natural sounding, such experiments are always vulnerable to the criticism that there may be something unnatural about the stimuli, so that, although the results are interesting psychophysically, they tell us little about speech perception. Recently, however, Cole, Cooper, Singer, and Allard (1975) have devised a technique whereby the shifting of perceptual boundaries can be demonstrated with stimuli made by splicing together recordings of naturally produced speech.

\section{Theoretical Considerations}

Most of the previous workers have implicitly, if not explicitly, followed Eimas and Corbit (1973) in supposing that the mechanism underlying selective adaptation is the fatiguing of feature detectors. The feature detectors almost certainly consist of neurons, or groups of neurons, probably located in the auditory cortex. Evans (1971), using microelectrode techniques, has reported neurons in the auditory cortex of cat which respond vigorously to an auditory stimulus at first, but which respond less reliably when the stimulus is repeated many times. It is most likely that similar units occur in the auditory cortex of man.

In vision, several types of adaptation effects have been isolated. Some of these, such as figural and movement aftereffects, decay with time and are probably explained by fatigue or saturation of feature detectors. In others, the effect is more persistent. In the McCollough effect (McCollough, 1965), for example, the association of line orientation and color persists seemingly indefinitely provided the eye is kept in total darkness, but decays on exposure to light (MacKay \& MacKay, 1975). Such cases are more readily explained by a structural change.

At present it is not known whether speech adaptation effects are the result of fatiguing the feature detectors or of some structural change. Cole et al. (1975), however, consider the latter to be unlikely, as they observed significant adaptation along a / / $\mathrm{ja} /-/ \mathrm{da} /$ dimension with a random series of adapting syllables in which the amount of frication varied from 25 to 60 msec.

In order to examine this question more directly, two possible mechanisms of adaptation will be considered. These are illustrated in Figure 1. Suppose that the speech recognition system contains two feature detectors, FD1 and FD2, which will respond differentially to an incoming signal, $S(x)$. Suppose that FD1 responds maximally when $x$, the parameter of $S(x)$, is equal to $x_{1}$ and FD2 responds maximally when $x$ is equal to $x_{2}$. At other values of $x$, the outputs of the feature detectors are lower, as shown in Figure 1.

The outputs of these detectors are connected to a further processor, which decides whether the incoming signal, $S(x)$, contained the feature represented by FD1 or the feature represented by FD2, depending on which detector has the greatest output. This model is similar to that proposed by Miller (1975), who showed that the relative strength of the output of a detector is registered by the higher centers of processing. The perceptual boundary, $B_{12}$, will lie at the point where the strengths of the outputs of the two detectors are equal.

If FD1 is fatigued by the repetition of a signal $S\left(x_{1}\right)$, the strength of its output will be reduced, as shown in Figure 1a. The boundary, $B_{12}$, where the response strengths are equal, will shift towards $x_{1}$, as shown.

Although the position of the boundary can be determined by experiment, the exact value of $x_{1}$ is unknown. It could be that in an experiment the adapting signal was $S\left(x_{1}{ }^{\prime}\right)$, where $x_{1}{ }^{\prime}$ differs from $x_{1}$ by a small amount. The result of repetition might be to "retune" FD1 so that its response area shifted so that it responded maximally to $S\left(x_{1}{ }^{\prime}\right)$. This would cause a shift in the boundary, $B_{12}$, as shown in Fig-
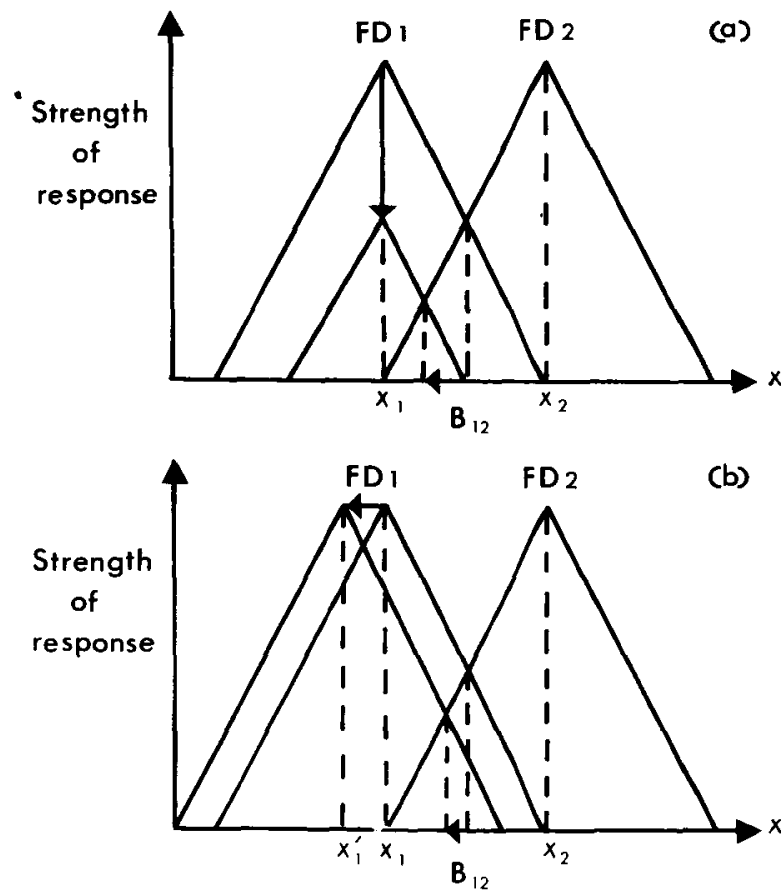

Figure 1. Strength of response of two feature detectors, FD1 and FD2, as a function of the signal S(x). (a) Fatigue hypothesis: Adaptation reduces the output of FD1 causing the perceptual boundary $B_{12}$ to shift. (b) Retuning hypothesis: Adaptation retunes FD1 so that its strength of response is greatest to a signal $S\left(x_{1}{ }^{\prime}\right)$, with a resultant shift in $B_{12}$. 
ure $1 \mathrm{~b}$. This is similar in direction to the boundary shift produced by the fatigue mechanism.

Although the two mechanisms might lead to similar boundary shifts when the adapting syllables are taken from the ends of the test series, they lead to different predictions with adaptation by a signal $S(x)$ when $x$ has values between $x_{1}$ and $x_{2}$.

The fatigue hypothesis predicts that when $x$ has the value $x_{1}$, the boundary $B_{12}$ will be shifted towards $x_{1}$. As the value of $x$ in the adapting signal $S(x)$ is increased in the direction of $x_{2}$, the fatigue hypothesis predicts that the shift in boundary will decrease, become zero when $x$ has a value near to the unadapted boundary, then increase in the other direction, reaching a maximum when $x$ is equal to $x_{2}$ (Figure 2a). The boundary shift will be a continuous function of the parameter of the adapting signal.

The retuning hypothesis leads to a different prediction. Let us suppose that the retuning takes place in the direction of the value of $x$ in the adapting signal $\mathrm{S}(\mathrm{x})$. For values of $\mathrm{x}$ less than $\mathrm{x}_{1}, \mathrm{FD} 1$ will be retuned and the boundary will shift in the direction of $x_{1}$ (Figure 2b). When $x$ has a value equal to $x_{1}$, there will be no shift in the boundary. For values just greater than $x_{1}$, there will be a shift towards $x_{2}$ from the unadapted position.

When the value of $x$ is on the other side of the unadapted boundary, FD2 will be retuned; when $x$ is less than $x_{2}$, the boundary will shift towards $x_{1}$. When $x$ has a value equal to $x_{2}$, there will be no shift from the unadapted position, and beyond $x_{2}$, the boundary will shift in the direction of $x_{2}$. The retuning hypothesis thus predicts a reversal in the boundary shift between $\mathrm{x}_{1}$ and $\mathrm{x}_{2}$.

The predictions of the two hypotheses are thus quite different in the region between $x_{1}$ and $x_{2}$. If the adapting signal $S(x)$ is given a series of values in the region between $x_{1}$ and $x_{2}$, and the position of the adapted boundary $B_{12}$ is determined, the fatigue hypothesis predicts that the shift of the boundary will increase continuously with $x$ on either side of the unadapted boundary, while the retuning hypothesis predicts a reversal of shift in that region.

\section{EXPERIMENTS}

In order to test the validity of the hypotheses, a series of experiments were performed in which listeners were asked to identify synthetic syllables as $/ \mathrm{di} /, / \mathrm{ti} /$, or $/ \mathrm{si} /$. The duration of frication was varied so that good exemplars of these syllables were included in the stimulus series. In this way, the positions of two perceptual boundaries, $/ d /-/ t /$ and $/ t /-$ $/ \mathrm{s} /$, were determined for a series of adaptation conditions.

\section{Method}

Subjects. Six unpaid volunteers acted as listeners in the experiment. They were staff (A, B, and C) and students (D, E, and F)

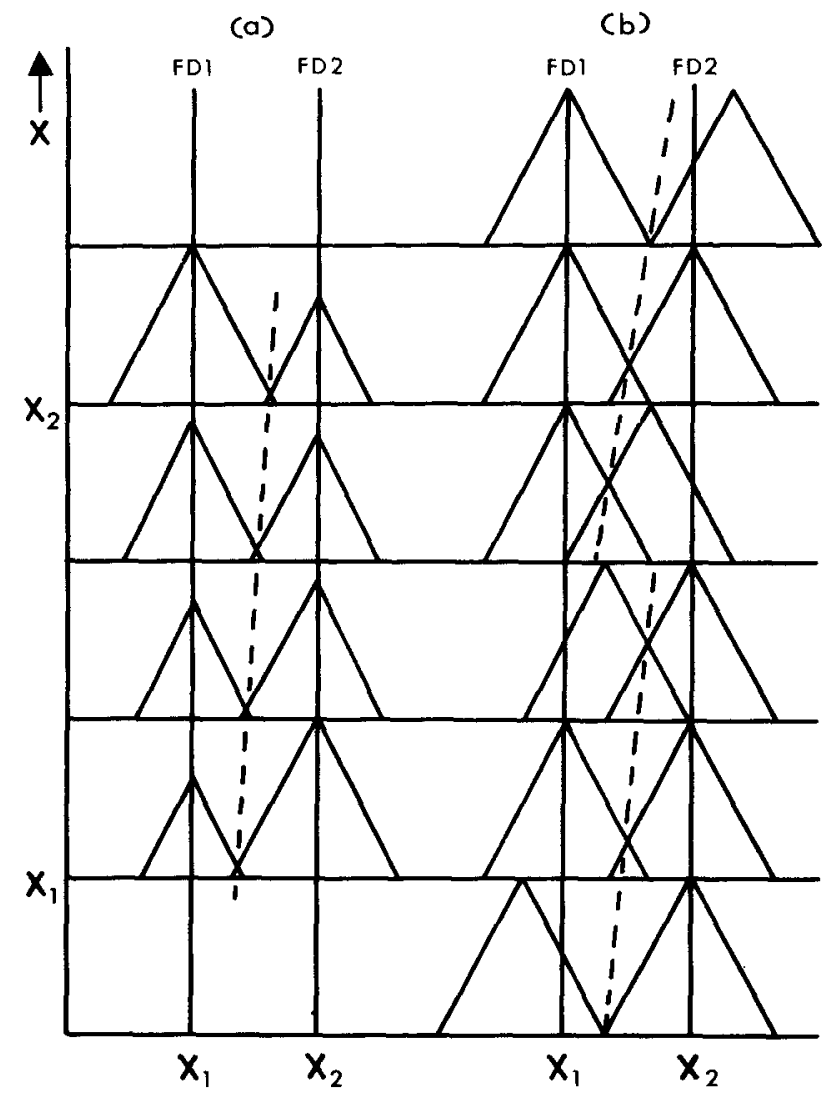

Figure 2. Shift of the boundary $B_{12}$ as a function of $x$, the parameter of the adapting signal $S(x)$, according to (a) the fatigue hypothesis, and (b) the retuning hypothesis. The position of the boundary is shown by the dashed lines.

of the University of Keele. The staff were familiar with synthetic speech, but the students were not.

Stimuli. The stimuli were speech patterns generated by a parallel resonance speech synthesizer (Holmes, Mattingly, \& Shearme, 1964) controlled by a digital computer (Ainsworth \& Millar, 1971). Each stimulus consisted of a burst of noise in the band 4 to $5 \mathrm{kHz}$ followed by a three-formant voiced sound. The frequencies of the formants were $220,2,560$, and $3,340 \mathrm{~Hz}$. The duration of the burst of noise was one of the values in the series $10,20, \ldots$ $160 \mathrm{msec}$, while the duration of the voiced sound remained constant at $250 \mathrm{msec}$. The fundamental of the voiced part of the sound fell linearly from 120 to $80 \mathrm{~Hz}$.

Apparatus. The stimuli were presented on-line in a quiet room remote from the computer. The signals from the synthesizer were amplified by a Revox G36 tape recorder, and presented via a Celestion Ditton 10 loudspeaker in the ceiling of the quiet room.

The responses were recorded on-line. Listeners were asked to press one of the switches on a box in front of them. The switches were labeled $D, T, S$, and OTHER. The computer waited for all listeners to respond before generating the next stimulus. All of the listeners took part simultaneously.

Procedure. The positions of the unadapted boundaries were established first. Sixteen stimuli with frication durations in the series $10,20, \ldots 160 \mathrm{msec}$ were presented in random order. This was repeated 10 times, with a different order on each occasion.

The positions of the boundaries after various conditions of adaptation were established next. The procedure was as before, but each test syllable was preceded by an adapting syllable repeated 32 times at a rate of once every $1 / 2 \mathrm{sec}$. The subjects were instructed to listen to the entire sequence but to respond only to the consonant in the last syllable. 
Four randomized sequences of 16 test syllables were presented in each session, the adapting syllable being kept constant for an entire session. Sessions took place at weekly intervals.

Seven adapting syllables were employed with frication durations having values of $20,40, \ldots 140 \mathrm{msec}$. These were randomized between the sessions. Four of the listeners heard each sequence 8 times, and two of them 16 times.

\section{Results}

The perceptual boundaries were determined by two methods. First, the numbers of responses in each category were plotted against frication duration, as shown for the unadapted boundaries of one of the listeners in Figure 3. The points at which there were $50 \%$ of the responses in each category were estimated by linear interpolation. This gave a good measure of the positions of the boundaries, but with the small amount of data it was difficult to estimate the accuracy.

Second, the data for each run of 16 values was examined. In most instances it was possible to place the boundary to within $10 \mathrm{msec}$. In cases where there was some confusion at the boundary, the area of confusion was delimited and the boundary was placed at the center of this area. The means and standard deviations for these boundaries were calculated. The means are shown by the arrows in Figure 3, and are in excellent agreement with the boundaries determined by the $50 \%$ criterion.

The mean standard deviation for all runs with all listeners was $6.8 \mathrm{msec}$ (range 4.0 to $8.4 \mathrm{msec}$ ). With eight runs, differences of $5 \mathrm{msec}$ or more are significant at the $5 \%$ level, and for 16 runs, differences of $3 \mathrm{msec}$ are significant at the $5 \%$ level.

The positions of the perceptual boundaries as a function of the duration of frication in the adapting syllable are shown for each listener in Figure 4. The results of listeners $A$ and $B$ were based on 16 runs, and the rest on 8 runs each.

The positions of the unadapted boundaries vary from listener to listener, but are approximately at $40 \mathrm{msec}$ for the $/ \mathrm{d} /-/ \mathrm{t} /$ boundary and $100 \mathrm{msec}$ for the $/ t /-/ s /$ boundary. This suggests that the feature detector responsible for recognizing $/ t /$ was tuned to about 70 msec.

The retuning hypothesis predicts discontinuities in the adapted boundary. There is no indication that this hypothesis is supported.

The fatigue hypothesis predicts that the adapted $/ d /-/ t /$ boundary should rise in the region immediately before $70 \mathrm{msec}$, and the $/ \mathrm{t} /-/ \mathrm{s} /$ boundary should rise in the region about $70 \mathrm{msec}$. This prediction is fulfilled for all listeners in both cases.

\section{Discussion}

Although the fatigue hypothesis seems to be well supported by the data, there are several unexpected points which have arisen. First of all, the obtained

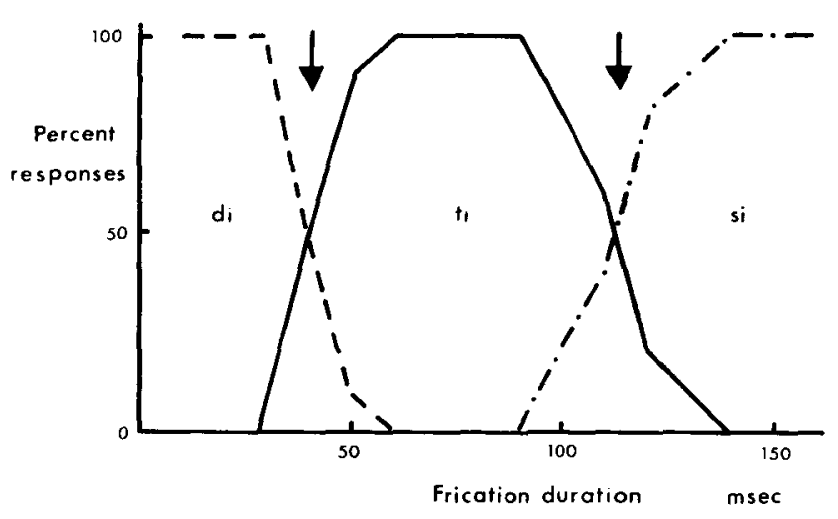

Figure 3. Percent responses of $/ d /, / t /$, and $/ s /$ as a function of frication duration for one listener. The arrows show the mean boundaries from individual runs (see text).

boundary curves are not quite as predicted in Figure 2. It was expected that the $/ \mathrm{t} /-\mathrm{s} / \mathrm{s}$ boundary curve below the /t/ value (about $70 \mathrm{msec}$ ) would return to the unadapted boundary. This clearly does not happen. In all cases, the boundary continues to deviate from the unadapted value in an approximately linear fashion. In one case (subject $\mathrm{E}$ ), there is a suggestion that the boundary begins to return to the unadapted position at about $30 \mathrm{msec}$.

One possible explanation is that the retuning mechanism is operative as well as the fatigue mechanism, and dominates the boundary in this region. It would be expected, however, that the retuning mechanism would operate on the $/ d /-/ t /$ curve in a similar fashion and cause it to rise in the region above $70 \mathrm{msec}$. The data do not support this suggestion.

An alternative explanation is that the experiments did not involve a trimodal feature detector corresponding to $/ d /, / t /$, and $/ s /$, but a pair of bimodal detectors, one of which responded to the features "voiced" and "voiceless" and was responsible for the lower boundary curve and one of which responded to the features "stop" and "fricative" and was responsible for the upper curve. In the case of this latter detector, it would not matter whether the incoming signal was $/ d /$ or $/ t /$. Either would fatigue the detector and cause the extended deviation from the unadapted boundary.

It is not known whether the adaptation in the present experiments involved acoustic or linguistic feature detectors. It may be that the "voiced/voiceless" detector simply distinguished sounds with frication duration in the range $0-40 \mathrm{msec}$ from those with it in the range 40-80 msec, and the "stop/fricative" detector distinguished sounds with frication duration less than 100 msec from those with it greater than 100 msec.

A further unexpected result was that in some cases the $/ \mathrm{t} /-/ \mathrm{s} /$ boundary curve did not pass through the 


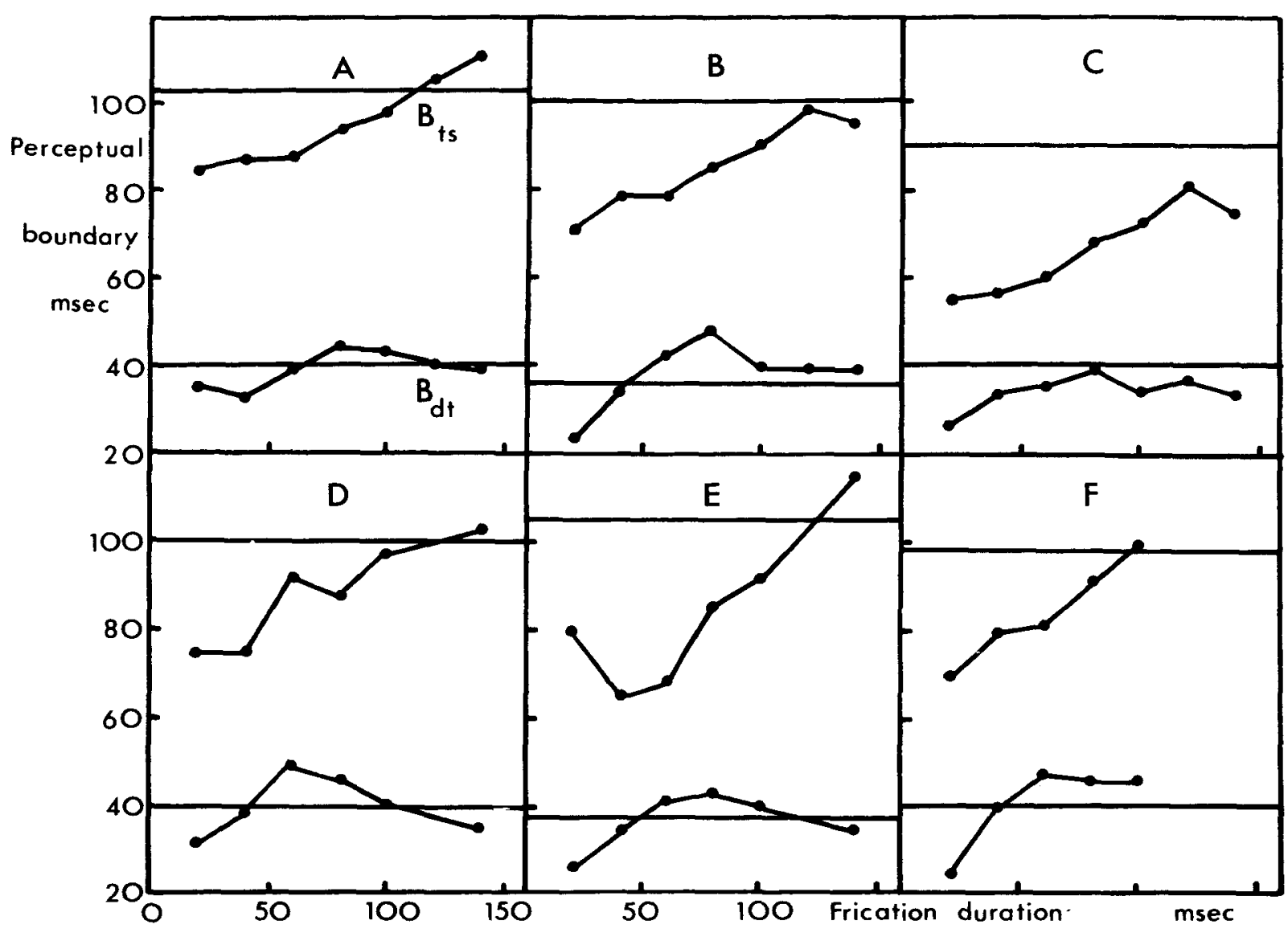

Figure 4. Perceptual boundaries as a function of frication duration in the adapting syliable. Horizontal lines are the positions of the unadapted boundaries.

unadapted boundary value. This suggests that adaptation with a stimulus lying on the unadapted boundary may cause a shift in the boundary. This is, of course, possible if one of the feature detectors is more sensitive to fatigue than the other. In the case above, the /t/ or "stop" detector is more readily fatigued than the /s/ or "fricative" detector.

The suggestion that some feature detectors may be more easily fatigued than others is consistent with the symmetrical boundary shifts which have often been reported in the literature (Cole et al., 1975).

\section{CONCLUSIONS}

Two mechanisms of selective adaptation have been considered, and an experiment has been performed which was designed to distinguish between them. The shift in perceptual boundary was measured as a function of frication duration in the series $/ \mathrm{di} /-/ \mathrm{ti} /-/ \mathrm{si} /$. It was found that in certain regions the boundary shift was a linear function of frication duration, as predicted by the fatigue hypothesis.

The obtained shifts were consistent with a model consisting of two pairs of feature detectors, a "voiced/ voiceless" detector which was responsible for the $/ \mathrm{d} /-/ \mathrm{t} /$ boundary and a "stop/fricative" detector responsible for the $/ t /-/ s /$ boundary.

The results also suggested that feature detectors are not all equally sensitive to fatigue.

\section{REFERENCES}

ADEs, A. E. Bilateral component in speech perception? Journal of the Acoustical Society of America, 1974, 56, 610-616.

Answ ORTH, W. A. Some effects of adaptation on phoneme boundaries. In Proceedings of the Eighth International Congress of Phonetic Sciences, Leeds, 1975.

Answorth, W. A., \& Millar, J. B. A simple time-sharing system for speech perception experiments. Behavior Research Methods \& Instrumentation, 1971, 3, 21-24.

BAILEY, P. J. Perceptual adaptation for acoustical features in speech. In Speech Communication Seminar, Stockholm, 1974, $3,47-53$.

Cole, R. A., Cooper, W. E., Surger, J., \& Allard, F. Selective adaption using real speech. Perception \& Psychophysics, 1975, 18, 227-244.

COOPER, W. E. Adaptation of phonetic feature analyzers for place of articulation. Journal of the Acoustical Society of America, 1974, 56, 617-627.

DIEHL, R. L. The effect of selective adaptation on the identification of speech sounds. Perception \& Psychophysics, 1975, 17, 48-52. Ermas, P. D., Cooper, W. E., \& Corart, J. D. Some properties of 
linguistic feature detectors. Perception \& Psychophysics, 1973, 13, 247-252.

Ermas, P. D., \& Corbir, J. D. Selective adaptation of linguistic feature detectors. Cognitive Psychology, 1973, 4, 99-109.

Evans, E. F. Central mechanisms relevant to the neural analysis of simple and complex sounds. In Pattern recognition in biological and technical systems. Heidelberg: Springer, 1971. Pp. 327-343.

GANONG, W. F. An experiment on "phonetic adaptation." MIT Quarterly Progress Report, 1975, 116, 206-210.

holmes, J. N., Mattingly, I. G., \& Shearme, J. N. Speech synthesis by rule. Language and Speech, 1964, 7, 127-143.

Jakobson, R., Fant, G. C. M., \& Halle, M. Preliminaries to speech analysis. Cambridge, Mass: MIT Press, 1963.

Lisker, L., \& Abramson, A. S. Some effects of context on voice onset time in English stops. Language and Speech, 1967, 10, $1-28$.

MacKay, D. M., \& MACKAY, V. What causes decay of pattern- contingent chromatic aftereffects? Vision Research, 1975, 15, 462-464.

McCollougr, C. Colour adaptation of edge detectors in the human visual system. Science, 1965, 149, 1115-1116.

Mrlle R, J. L. Properties of feature detectors for speech: Evidence from the effects of selective adaptation on dichotic listening. Perception \& Psychophysics, 1975, 18, 389-397.

PIsonI, D. B. Stages of processing in speech perception. In Proceedings of the Eighth International Congress of Phonetic Sciences, Leeds, 1975.

Studdert-Kennedy, M., \& Shankweiler, D. Hemispheric specialization for speech perception. Journal of the Acoustical Society of America, 1970, 48, 579-594.

(Received for publication August 8, 1976; revision accepted January $15,1977$. 